\title{
Clinical and Plan Complexity Effects of Using Direct Aperture Optimization in Breast, Head and Neck IMRT Treatment
}

\author{
M. I. El Gohary, G. M. Kamal", M. M. Galal ${ }^{* *}$ and M. \\ Hosini $^{* * * \#}$ \\ Biophysics Department, Faculty of Science, AlAzhar University \\ (Boys); "Biophysics Department, Faculty of Science, AlAzhar \\ University (Girls); ${ }^{* *}$ Radiation Oncology Department \\ (NEMROK), Faculty of Medicine, Cairo University, Cairo and \\ ***Radiation Oncology Department, Faculty of Medicine, \\ Zagazig University, Zagazig, Egypt.
}

$\mathbf{T}$

O INVESTIGATE the planning complexity and dosimetric impacts of direct aperture optimization (DAO) in IMRT treatment of breast, head and neck $(\mathrm{H} / \mathrm{N})$ cancers.

Three breast and five H/N patients were planned using the beamlet optimizer in Elekta-Xio ${ }^{\odot}$ ver 4.6 IMRT treatment planning system. Based on our experience in beamlet IMRT optimization, breast PTVs were prescribed to $50 \mathrm{~Gy}$ with 2 fields. While PTVs in $\mathrm{H} / \mathrm{N}$ plans were prescribed to 70 Gy delivered by 7 fields.. In all plans, fields were set to be equally spaced. All cases were re-planed using Direct Aperture optimizer (DAO) in Prowess Panther ${ }^{\odot}$ ver 5.01 IMRT planning system at same configurations and dose constraints. Plans were evaluated according to ICRU criteria, number of segments, number of monitor units and planning time.

For H/N plans, the dose that covers $95 \%\left(D_{95}\right)$ from PTV was $0.8 \%$ on average for plans done using beamlet optimizer more than that done using DAO, while D98\%, D50\% and D2\% were higher in DAO plans than in beamlet optimizer by about $3 \%, 1 \%$, and $2.2 \%$, respectively. For OAR, results showed an improvement in lung sparing in plans done using beamlet optimizer where V20 was lower by $12 \%$. While DAO improved heart sparing than beamlet optimizer as V20 was lower $12 \%$. These results achied using around less $45 \%$ segments number and $50 \%$ monitor units number as well.

In DAO $\mathrm{H} / \mathrm{N}$ plans, the near maximum dose $\left(\mathrm{D}_{2}\right)$ and the dose that covers $95 \%\left(\mathrm{D}_{95}\right)$ of PTV has improved by $4 \%$ in DAO. For organs at risk (OAR), DAO reduced the volume covered by $30 \%\left(\mathrm{~V}_{30}\right)$ inspinal cord, right parotid, and left parotid by 60\%, 54\%, and 53\% respectively. This considerable dosimetric quality improvement achieved using $25 \%$ less planning time and lower number of segments and monitor units by $46 \%$ and $51 \%$, respectively.

\footnotetext{
\#Corresponding author e-mail : $\underline{\text { mhosini@ksu.edu.sa }}$
} 
DAO introduces considerable advantages over beamlet optimization in regards to organ at risk sparing. While no significant improvement occurred in the PTV ICRU reporting dose.

Keywords: DAO, IMRT, Optimization.

Most of the IMRT plans are done with beamlet-based inverse planning methods ${ }^{(1-10)}$, where by the optimization algorithm optimizes the intensities of finite- sized pencil beams (beamlets) that make up each treatment beam irradiating the patient. A leaf-sequencing algorithm then translates the intensity "map" into segmented fields that can be delivered by a multileaf collimator (MLC) ${ }^{(11-14)}$. Although it is the most widely used approach to IMRT, this method has limitations. The segmentation usually results in too many small segments that require large monitor units. This means a large leakage dose to the patient, long treatment time, and increased maintenance costs for the MLCs. In addition, dosimetry of the plan is compromised by the segmentation process, owing to the restrictions on the number of intensity levels set by the user and machinespecific limitations on MLCs.

In this work, we used a different IMRT optimization algorithm, direct aperture optimization (DAO), to plan IMRT for breast and head \&neck patients. Direct aperture optimization IMRT is an IMRT method in which the aperture shapes and aperture weights are optimized simultaneously, and the MLC constraints and the number of segments are directly included in the optimization process $^{(15)}$. In DAO-IMRT planning, the planner specifies the planning objectives on the basis of the dose volume criteria for the target and critical structures as well as the number of beam segments to be delivered. The optimization only considers aperture shapes that satisfy the conditions set by the MLC. As a result, high-quality DAOIMRT treatment plans can be generated using fewer segments (apertures) per beam. We compared the quality of treatment plans according to ICRU $83^{(16)}$ criteria, dose to the surrounding normal tissues of heart and lung, segments number, and the number of monitor units to be delivered.

Shepard et al. ${ }^{(15)}$ introduced the concept of DAO and showed that when applied to several patient cases, it resulted in highly conformal dose distributions with significantly fewer segments and monitor units (MUs) than conventional optimization methods. The DAO plans were generated using a noncommercial planning system with a Monte Carlo-based dose calculation.

Bergman et al. ${ }^{(17)}$ introduced a Monte Carlo-based DAO algorithm. For a nasopharynx case, they found approximately 33\% improvement in MU efficiency when the optimization engine was changed from two-step optimization to one-step optimization.

Several studies reported clinical comparisons of one-step and two-step optimization in the Pinnacle TPS. In this TPS, the one-step optimization is

Egypt. J. Biophys. Biomed. Engng. Vol. 17 (2016) 
referred to as the direct machine parameter optimization (DMPO), and the twostep method is referred to as the intensity modulation (IM). From a study of 11 head-and-neck plans, Jones and Williams ${ }^{(18)}$ found that fewer segments were used in DMPO plans than corresponding IM plans.

Marcello Sabatino ${ }^{(19)}$ studied difference between beamlet and DAO IMRT and found no superior system in terms of PTV coverage and OAR sparing. Major differences in efficiency of the method in terms of calculated MU and treatment times were found.

DMPO and IM IMRT plans were also compared by van Asselen et al. ${ }^{(4)}$ for twelve breast cancer patients. They found no significant reduction in MUs, but a reduction in the number of segments. As well, Ahunbay et al. ${ }^{(20)}$ compared the DAO method in the Panther TPS and the two-step optimization in the XiO CMS TPS for ten cases of whole breast treatment. They observed that the total number of MUs for DAO plans were approximately $60 \%$ less than those of two-step optimization IMRT plans.

\section{Materials and Methods}

We used the XiO Planning system (CMS, Elekta Inc.) for beamlet-IMRT planning and the Prowess Panther planning system (Prowess, Chico, CA) for our DAO-IMRT planning. All treatment plans were designed for delivery on a Siemens Oncor linear accelerator equipped with a80-leaf double focused MLC (Siemens Healthcare USA, Inc) using 6-MV photons delivered at $400 \mathrm{MU} / \mathrm{min}$ with a step-and-shoot IMRT method. Both the $\mathrm{XiO}$ and Prowess treatmentplanning systems use convolution-based dose calculation algorithms.

For each patient, a plan was generated firstly using the beamlet optimization method and then using DAO, wherever possible, identical parameters were used. These parameters included the number and direction of beams; which was determined experimentally by making plans for each patient on Xio TPS with different beam numbers and directions, then select the optimum plan (results not shown here). Also, the dose objectives and their relative weights were kept constant. The convolution dose calculation took place between the $5^{\text {th }}$ and the $8^{\text {th }}$ iteration.

For the segmentation, a minimum segment size of $1 \mathrm{~cm}^{2}$ and minimum MUs of 2 MU were specified. These parameters had been derived from previously published values and independently confirmed for use at our institution ${ }^{(21)}$.

Seven intensity levels per beam were used for the beamlet intensity based optimization. This was consistent with the findings of Keller-Reichenbecher et al. ${ }^{(22)}$, which established that using between 5 to 7 intensity levels was sufficient for most IMRT treatments. For the plans using DAO value of segments per beam was determined experimentally (results not shown). In DAO, the convolution dose iteration is also the point at which the optimized intensity map is converted into MLC segments for the first time. 
For breast cases all plan objectives were; the prescribed dose was 50Gy, at least $95 \%$ of the prescribed dose covers $100 \%$ of PTV volume prescription dose, and keep the amount of volume of the PTV at $52 \mathrm{~Gy}$, which is $\sim 105 \%$ of the prescribed dose, close to zero.

For head and neck cases all plan objectives were; the prescribed dose was $70 \mathrm{~Gy}$, at least $95 \%$ of the prescribed dose covers $100 \%$ of PTV volume prescription dose, and keep the amount of volume of the PTV at $72 \mathrm{~Gy}$, which is $\sim 103 \%$ of the prescribed dose, close to zero,

Plans were evaluated based on ICRU 83 criteria (D98, D95,D50, D2, and V30), number of segments, number of monitor units and planning time. The results for 3 breast and 5 head and neck cases have been summarized.

\section{Results}

For breast plans, optimum plans done using beamlet optimizer were compared with those done using DAO, where doses delivered to target volumes and OAR were measured, and also comparing the number of segments and total number of monitor units should be delivered from clinical linear accelerator.

The results showed, for PTV, there was no significant difference between DAO and beamlet in all plans in $95 \%$ isodose coverage. The average difference in GTV volume which was covered by the prescribed dose (V100) was 3\% on average in plans done using beamlet optimizer more than those done using DAO. And D2\% was higher in DAO optimizer plans than in beamlet optimizer by about $2.5 \%$. Figure 1 showed GTV average dose differences between DAO and beamlet optimizer.

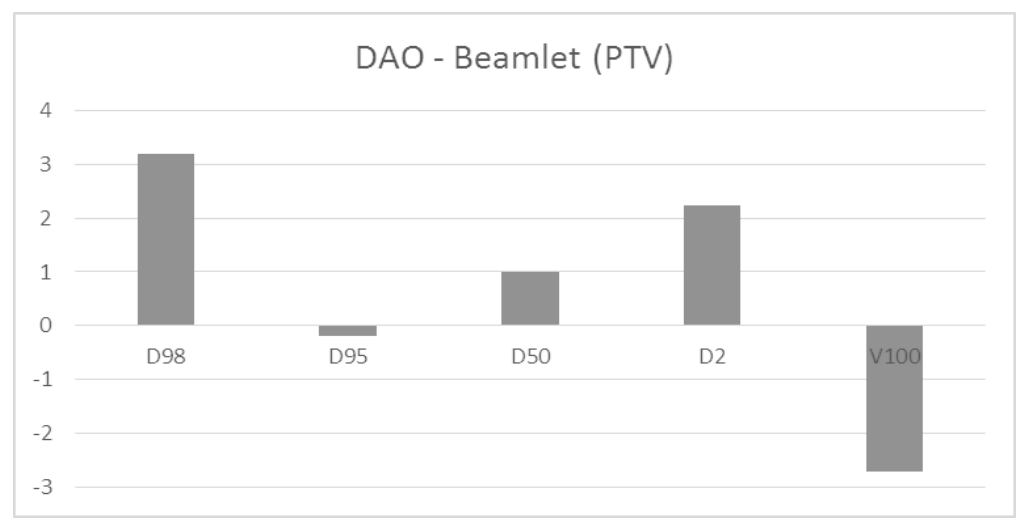

Fig. 1. The graph of GTV dose coverage difference between DAO and beamlet optimizer plans for breast case.

Egypt. J. Biophys. Biomed. Engng. Vol. 17 (2016) 
For OAR, For OAR, results showed an improvement in lung sparing in plans done using beamlet optimizer where D50\%, D2\%, and V20 were lower by $0.8 \%$, $3.5 \%$, and $12.1 \%$ respectively. While DAO improved heart sparing than beamlet optimizer as D50\%, D2\%, and V20 were lower by 9\%, 25\%, and $12 \%$ respectively. Figure 2 showed the dose difference between DAO and beamlet plans for OAR.

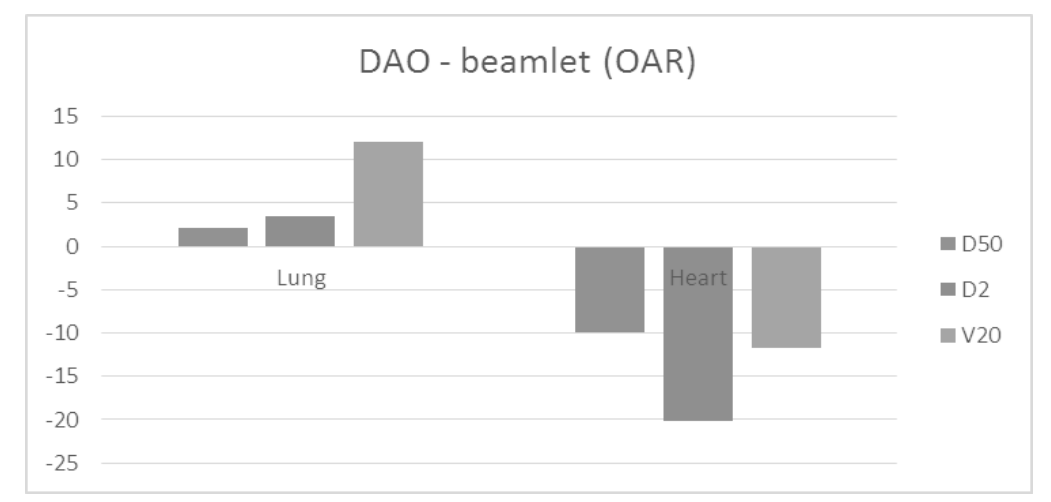

Fig. 2. The graph of OAR dose coverage difference between DAO and beamlet optimizer plans for brast case.

The number of segments in plan using DAO was constant at 14 because the planner determine it by himself, while it was varies from 25 up to 31 segments in plan using beamlet optimizer as shown in Fig. 3.

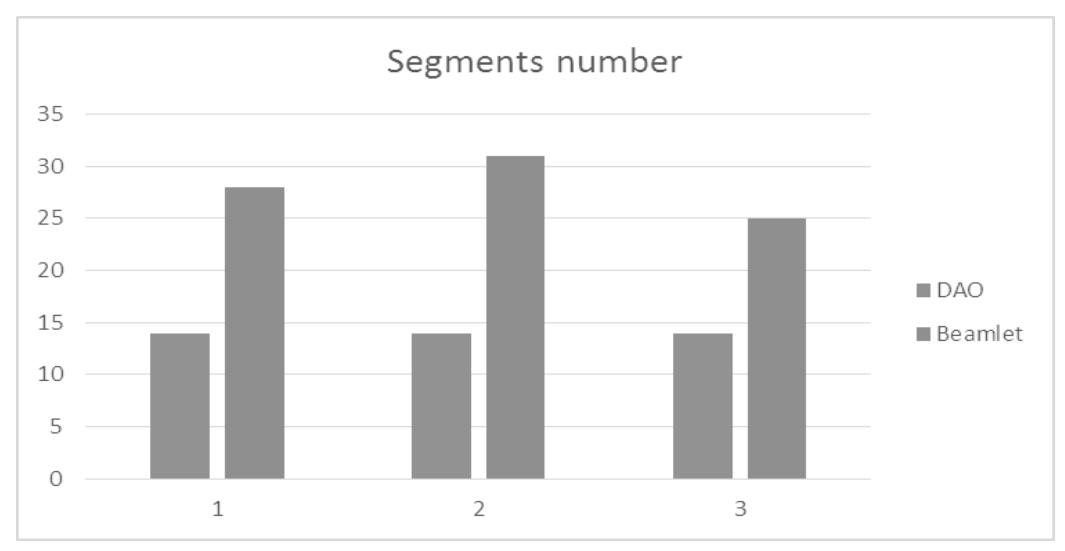

Fig. 3. The graph of segments number for breast cases planned using DAO and beamlet optimizer plans.

Total monitor units should be delivered from linear accelerator was lower in DAO plans than those for beamlet plans by about 52\% on average as shown in Fig. 4. 


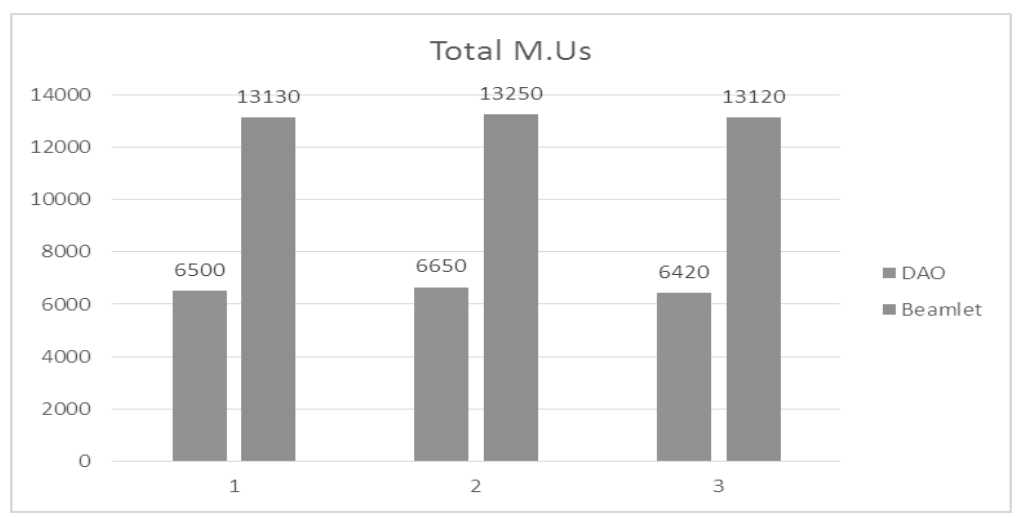

Fig. 4. The graph of total number of monitor units for breast cases planned using DAO and beamlet optimizer plans.

For $\mathrm{H} / \mathrm{N}$ plans, the optimum plans done using beamlet optimizer was compared with those done using DAO based on criteria defined by ICRU 83, where doses delivered to target volumes and OAR were measured, and also comparing the number of segments and total number of monitor units should be delivered from clinical linear accelerator.

As shown from the results, for GTV the average percentage difference for all cases in $95 \%$ isodose coverage was $1 \%$ plans done using DAO more than that done using beamlet optimizer, the average difference in volume which was covered by the prescribed dose was $12 \%$ in plans done using DAO more than those done using beamlet optimizer, and the hot area was $4 \%$ more in beamlet plan than DAO plan. For PTV the average difference in $95 \%$ isodose coverage was $3 \%$ plans done using beamlet optimizer more than that done using DAO, the average difference in volume which was covered by the prescribed dose was $4 \%$ in plans done using beam optimizer more than those done using DAO, and the hot area was $4 \%$ more in beamlet plan than DAO plan. Figure 5 showed GTV and PTV average dose difference between DAO and beamlet optimizer.

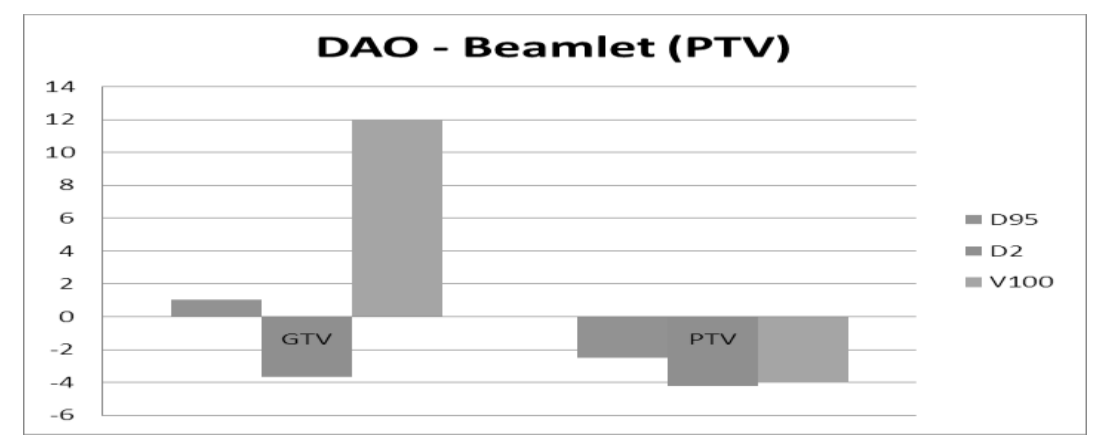

Fig. 5. The graph of GTVs and PTVs dose coverage difference between DAO and beamlet optimizer plans.

Egypt. J. Biophys. Biomed. Engng. Vol. 17 (2016) 
For OAR, results showed an improvement in OAR sparing up to more than $27 \%, 22 \%$, and $29 \%$ on average in rt. Parotid and 1t. parotid and spinal cord respectively in plans done using DAO, Figure 6 showed the dose difference between DAO and beamlet plans for OAR.

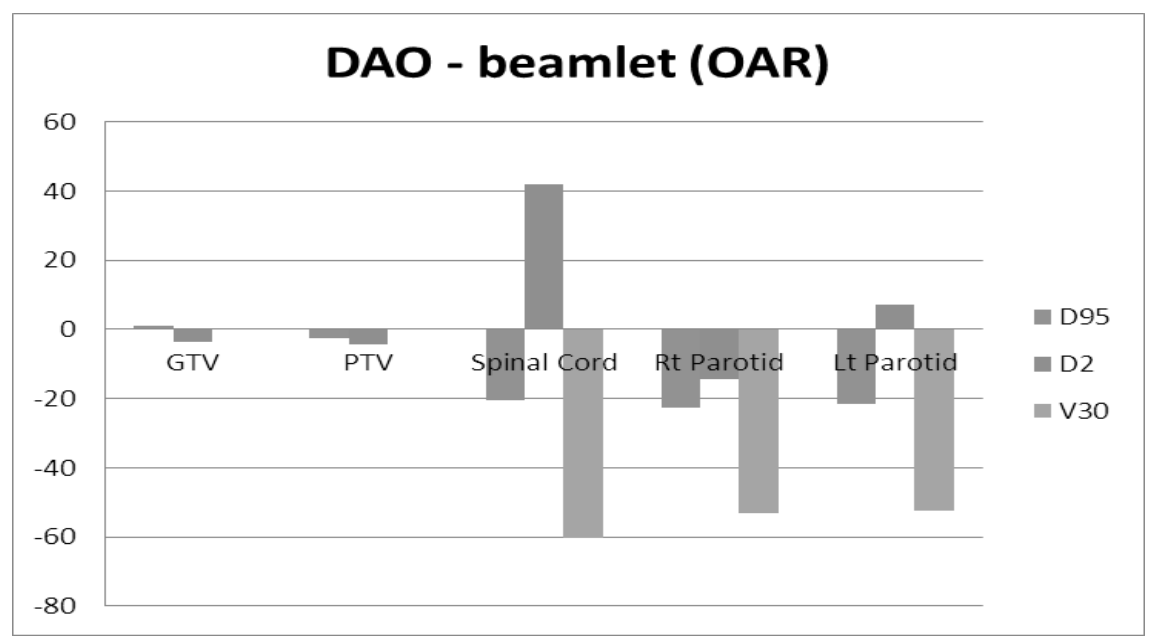

Fig. 6. The graph of OAR dose coverage difference between DAO and beamlet optimizer plans.

The number of segments in plan using DAO was constant at 49 because the planner determine it by himself, while it was varies from 71 up to 129 segments in plan using beamlet optimizer as shown in Fig. 3. And the number of total monitor units should be delivered from linear accelerator was lower in DAO plans than those for beamlet plans by about $55 \%$ on average as shown in Fig. 7.

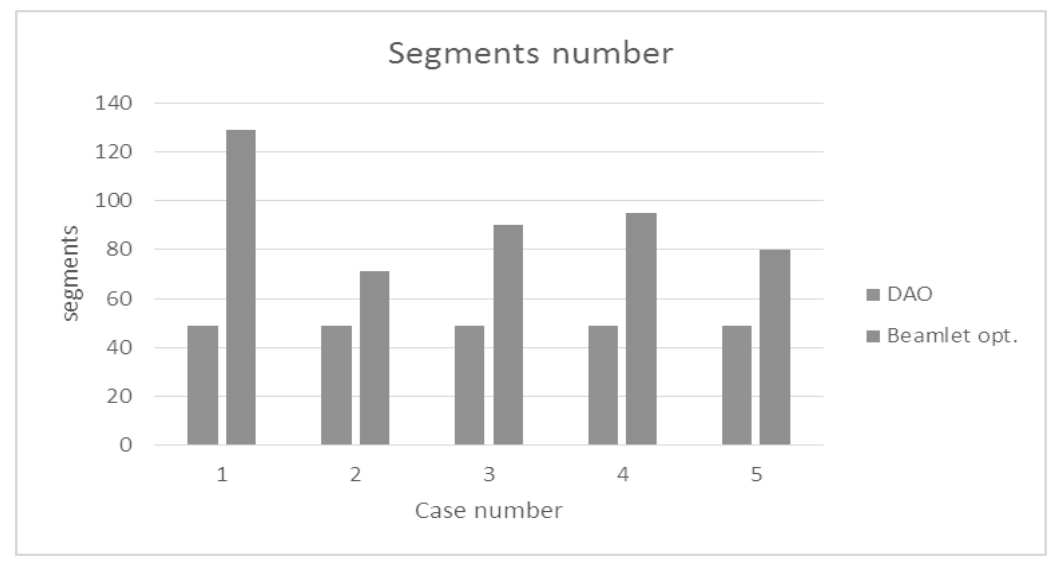

Fig. 7. The graph of segments number for DAO and beamlet optimizer plans. 


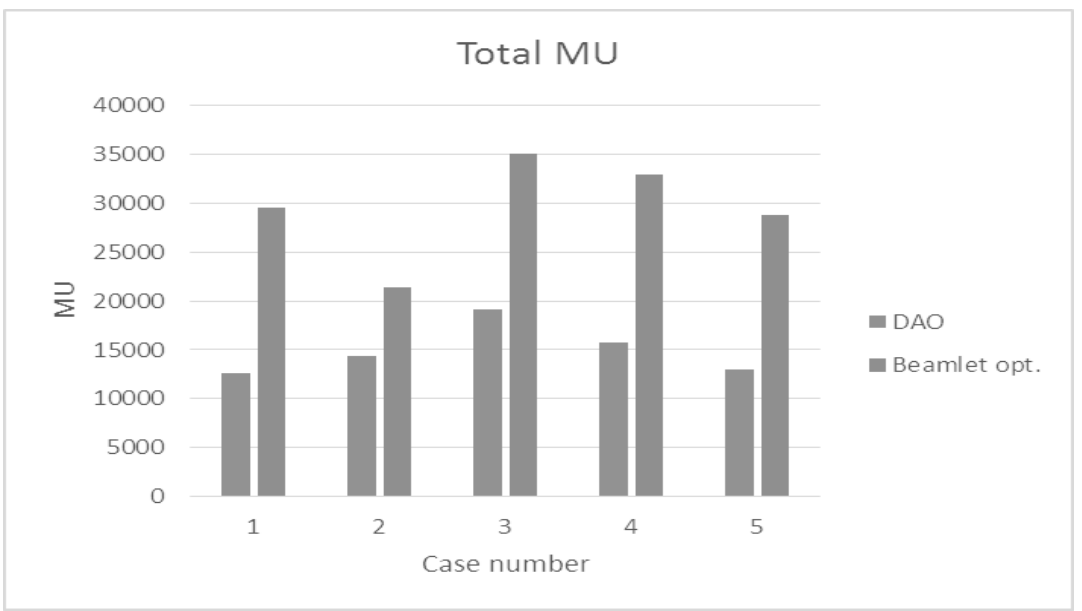

Fig. 8. The graph of total monitor units' number for DAO and beamlet optimizer plans.

\section{Discussion}

This study compares IMRT for breast, and head and neck tumors planned with different optimization methods. In terms of PTV coverage and OAR sparing all systems reach satisfactory and clinically acceptable results, even though some statistical significant differences can be observed.

This study showed that DAO IMRT plans and beamlet optimization demonstrated not significant difference in breast dose conformity $(0.5 \%)$. For OAR sparing the beamlet improved lung sparing better than DAO where difference in V20 lung dose was around 12\% \pm 0.3 . While DAO improved heart sparing as the difference in V20 heart dose was $6 \% \pm 5$. The MUs for DAO were approximately $60 \%$ less than those for beamlet IMRT. The number of segments for DAO was approximately $45 \%$ segments less than those for beamlet optimizer, this is in contrast to the findings of Van Asselen et al. ${ }^{(4)}$ who was the only group reported an increase in segments using DMPO for breast IMRT. In another study DAO-IMRT plans for 15 breast cancer patients in supine (10 patients) and prone (5 patients) positions with a goal of uniform dose coverage of the whole breast were designed ${ }^{(20)}$. These DAO-IMRT plans were compared with standard IMRT using beamlet optimization and conventional 3D-CRT plans using wedges. All plans used opposed tangential beam arrangements. Direct aperture optimized IMRT improved the overall quality of dose distributions as well as the planning and delivery efficiency for treating whole breast in both supine and prone positions. 
Head and neck the dose coverage has improved by $4 \%$ in DAO. For organs at risk (OAR), DAO reduced the volume covered by $30 \%\left(\mathrm{~V}_{30}\right)$ in spinal cord, right parotid, and left parotid by $60 \%, 54 \%$, and $53 \%$ respectively. DAO required lower number of segments and monitor units by $46 \%$ and $51 \%$ respectively. In another study of 10 hypopharyngeal patients, no statistically significant difference was found for compliance to the dose volume constraints although the mean dose to the parotid was lower with the beamlet based plans compared to the DAO plans. Dose homogeneity within the PTV was superior for the DAO plans and they also required significantly less MU to deliver ${ }^{(19)}$.

One of our goals from this study is the MU efficiency of the compared optimization algorithm. This is owed to the increasing number of MU in IMRT which could increase the risk of radiation induced secondary malignancies due to scattered radiation. Panther DAO plans resulted in decrease the amount of scatter radiation originating from the collimator head. Hall pointed out the need for protection of patients from scattered radiation in IMRT-treatments ${ }^{(23)}$. He reported a potential increase of radiation-induced cancer due to larger total body doses caused by leakage radiation. The reported reductions are in agreement with published studies.

Calculation time for all cases were also calculated in this study and it was found that the DAO IMRT treatments would easily fit into the about 18 - 25 min but the beamlet based IMRT treatments would require slightly longer treatment slots of $30-40$ min depending on target volume and the complexity of plan. the difference in calculation time arises from that beamlet perform calculations twice as it calculates the map intensity first and then recalculate to translate the map to deliverable segments while DAO perform the calculation once as discussed earlier.

\section{Conclusion}

DAO introduces considerable advantages over beamlet optimization in regards to organ at risk sparing. While no significant improvement occurred in the PTV ICRU reporting dose. The main advantage for using DAO is decreasing the number of segments to be used during treatment as well as decreasing the total number of monitor units should be delivered from linear accelerator which is reflecting on the treatment time and scattered radiation to the patients.

\section{References}

1. Mihai, A., Rakovitch, E. and Sixel, K., et al., Inverse vs. forward breast IMRT planning. Med. Dosim. 30, 149-154 (2005).

2. Hong, L., Hunt, M. and Chui, C., et al., Intensity-modulated tangential beam irradiation of the intact breast. Int. J. Radiat Oncol Biol Phys 44, 1155-1164 (1999).

3. Teh, B.S., Lu, H.H. and Sobremonte, S., et al., The potential use of intensity modulated radiotherapy (IMRT) in women with pectusexcavatum desiring breastconserving therapy. Breast J. 7, 233-239 (2001).

Egypt. J. Biophys. Biomed. Engng. Vol. 17 (2016) 
4. Van Asselen, B., Raaijmakers, C.P. and Hofman, P. et al., An improved breast irradiation technique using three-dimensional geometrical information and intensity modulation. Radiother, Oncol. 58, 341-347 (2001).

5. Partridge, M., Aldridge, S. and Donovan, E., et al. An intercomparison of IMRT delivery techniques: A case study for breast treatment. Phys Med. Biol. 46, 175-185 (2001).

6. Hurkmans, C., Cho, J. and Damen, E., et al., Reduction of cardiac and lung complication probabilities after breast irradiation using conformal radiotherapy with or without intensity modulation. Radiother, Oncol. 62, 163-171 (2002).

7. Cho, B.C., Hurkmans, C.W. and Damen, E.M. et al., Intensity modulated versus non-intensity modulated radiotherapy in the treatment of the left breast and upper internal mammary lymph node chain: A comparative planning study. Radiother Oncol 62, 127-136 (2002).

8. Lo, Y.C., Yasuda, G. and Fitzgerald, T.J. et al., Intensity modulation for breast treatment using static multi-leaf collimators. Int. J. Radiat. Oncol. Biol. Phys. 46, 187-194 (2000).

9. Vicini, F.A., Sharpe, M. and Kestin, L. et al., Optimizing breast cancer treatment efficacy with intensity-modulated radiotherapy. Int. J. Radiat. Oncol. Biol. Phys. 54, $1336-1344$ (2002).

10. Thatcher, S.H., Jursinic, P.A. and Yun, H. et al., Comparison of intensity modulated and 3D-conformal treatment breast plans with and without lumpectomy boost [abstract]. Int. J. Radiat. Oncol. Biol. Phys. 60, S394 (2004).

11. Bortfeld, T., Haler, D.H. and Waldron, T.J. et al., X-ray compensation with multileaf collimators. Int. J. Radiat. Oncol. Biol. Phys. 28, 723-739 (1994).

12. Galvin, J.M., Chen, X.G. and Smith, R.M., Combining multileaf field to modulate fluence distributions. Int. J.Radiat. Oncol. Biol. Phys. 27, 697-705 (1993).

13. Webb, S., Configuration options for intensity-modulated radiation therapy using multiple static fields shaped by a multileaf collimator. Phys. Med. Biol. 43, 241-260 (1998).

14. Webb, S., Configuration options for intensity-modulated radiation therapy using multiple static fields shaped by a multileaf collimator. II: Constraints and limitations on 2D modulation. Phys. Med. Biol. 43, 1482-1495 (1998).

15. Shepard, D.M., Earl, M.A. and Li, X.A. et al., Direct aperture optimization: A turnkey solution for step-and-shoot IMRT. Med. Phys. 29, 1007-1018 (2002).

16. International Commission on Radiation Units and Measurements 2010, "Prescribing, Recording, and Reporting Photon-Beam Intensity-Modulated Radiation Therapy (IMRT)”, Journal of the ICRU Vol 10 (1) Report 83 (2010). 
17. Bergman, A.M., Bush, K., Milette, M.P., Popescu, I.A., Otto, K. and Duzenli, C., Direct aperture optimization for IMRT using Monte Carlo generated beamlets. Med. Phys. 33 (10), 3666-79 (2006).

18. Ahunbay, E.E., Chen, G.P. and Thatcher, S., Direct aperture optimization-based intensity-modulated radiotherapy for 15 . whole breast irradiation. Int. J. Radiat. Oncol. Biol. Phys. 67 (4), 1248-58 (2007).

19. Sabatino, M., Kretschmer, M., Zink, K. and Würschmidt, F., The impact of direct aperture optimization on plan quality and efficiency in complex head and neck IMRT. Radiation, Oncology, 7, 7 (2012).

20. Ahunbay, E.E., Chen, G.P. and Thatcher, S. et al., Direct aperture optimizationbased intensity-modulated radiotherapy for 15 . whole breast irradiation. Int. J. Radiat. Oncol. Biol. Phys. 67 (4), 1248-58 (2007).

21. Lydon, J.M., Theoretical and experimental validation of treatment planning for narrow MLC defined photon fields. Phys. Med. Biol. 50,2701-14 (2005).

22. Keller-Reichenbecher, M.A., Bortfeld, T., Levegrun, S., Stein, J., Preiser, K. and Schlegel, W., Intensity modulation with the "step and shoot" technique using a commercial MLC: a planning study. Multileaf collimator. Int. J. Radiat. Oncol. Biol. Phys. 45,1315-1324 (1999).

23. Hall, E.J., Intensity-modulated radiation therapy, protons, and the risk of second cancers. Int. J. Radiat. Oncol. Biol. Phys. 65,1-7 (2006).

(Received 9/11/2015;

accepted 18/10/2016) 


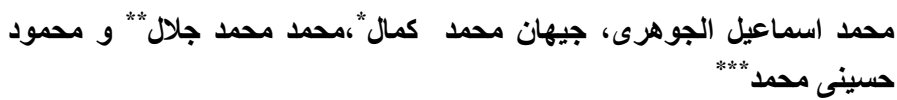

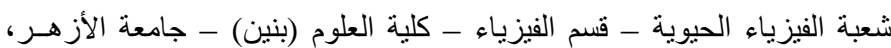

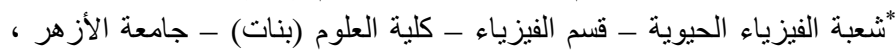

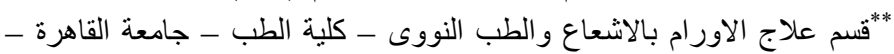

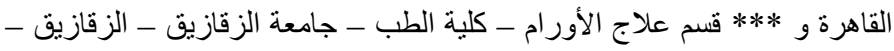

تم فى هذا البحث دراسة مدى تعقيد التخطيط وكذللك قياس الجرعة نتيجة الإستخدام

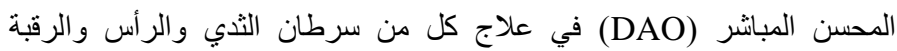

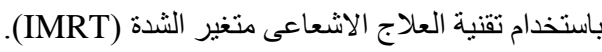

تم عمل التخطيط العلاجى لعدد ثلاثة حالات مصابة بسرطان الثدي وخمسة

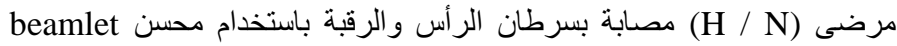

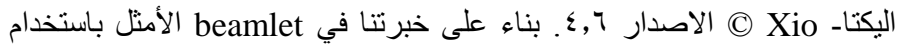

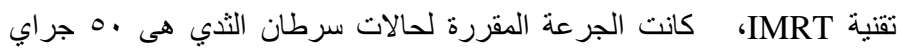

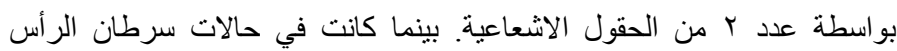

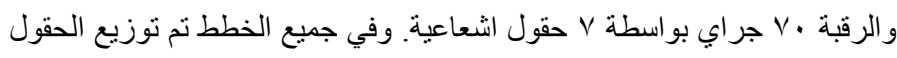

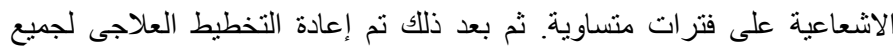

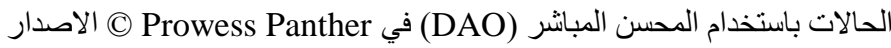

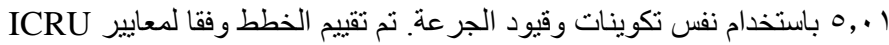

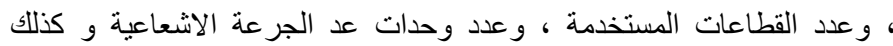

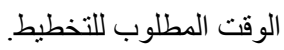

اوضحت النتائج انه بالنسبة لحالات سرطان الثذى، كانت الجرعة التي تغطي 90\%

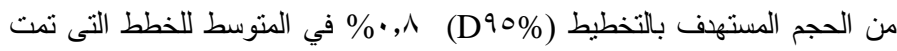

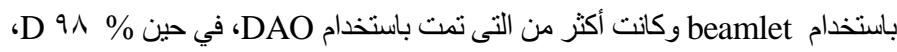

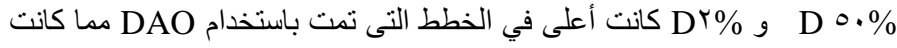

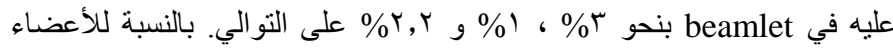
الخطرة (OAR)، فقد أظهرت النتائج تحسنا في تجنيب الرئة في الخطط باستخدام

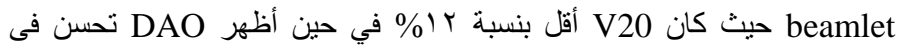

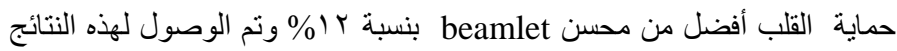

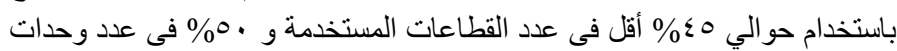

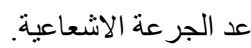

Egypt. J. Biophys. Biomed. Engng. Vol. 17 (2016) 
بالنسبة لحالات سرطان الرأس والرقبة ، كان الحد الأقصى للجرعة القريب

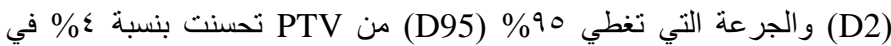

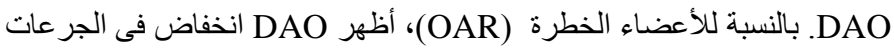

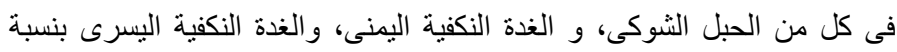

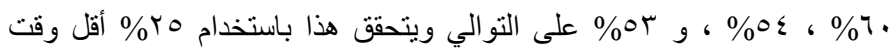

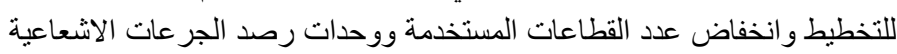

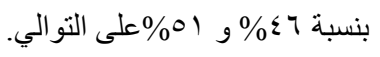

و قد تبين أن DAO يقدم مز ايا كبيرة على التحسين في في في في في في ما يخص حماية

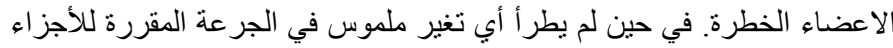

المستهدف علاجها. 